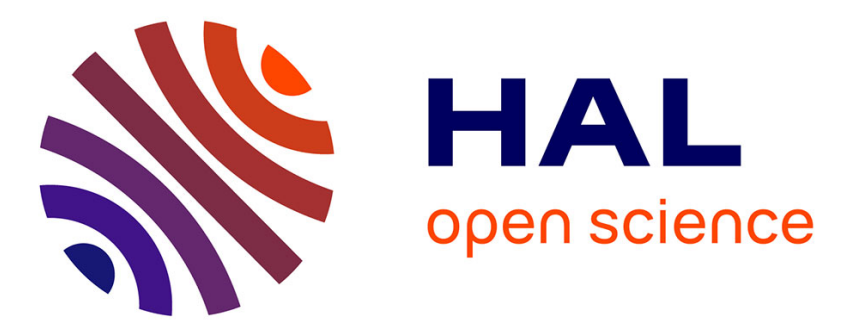

\title{
Magnetic properties of a fourfold degenerate state: $\mathrm{Np} 4+$ ion diluted in Cs2ZrCl6 crystal
}

\author{
Dayán P. Hernández, Hélène Bolvin
}

\section{To cite this version:}

Dayán P. Hernández, Hélène Bolvin. Magnetic properties of a fourfold degenerate state: Np4+ ion diluted in Cs2ZrCl6 crystal. Journal of Electron Spectroscopy and Related Phenomena, 2014, 194, pp.74-80. 10.1016/j.elspec.2014.03.002 . hal-01071980

\section{HAL Id: hal-01071980 \\ https://hal.science/hal-01071980}

Submitted on 3 Jun 2021

HAL is a multi-disciplinary open access archive for the deposit and dissemination of scientific research documents, whether they are published or not. The documents may come from teaching and research institutions in France or abroad, or from public or private research centers.
L'archive ouverte pluridisciplinaire HAL, est destinée au dépôt et à la diffusion de documents scientifiques de niveau recherche, publiés ou non, émanant des établissements d'enseignement et de recherche français ou étrangers, des laboratoires publics ou privés. 


\title{
Magnetic properties of a fourfold degenerate state: $\mathrm{Np}^{4+}$ ion diluted in $\mathrm{Cs}_{2} \mathrm{ZrCl}_{6}$ crystal
}

\author{
Dayán Páez Hernández, Hélène Bolvin* \\ Laboratoire de Chimie et de Physique Quantiques, IRSAMC, Université de Toulouse III, 118 route de Narbonne, 31062 Toulouse Cédex 04, France
}

\begin{abstract}
The magnetic properties of the octahedral cluster $\mathrm{NpCl}_{6}^{2-}$ diluted in $\mathrm{Cs}_{2} \mathrm{ZrCl}_{6}$ crystal have been calculated using a first principle method, SO-CASPT2. The spin Hamiltonian parameters modeling the fourfold degenerate ground state are extracted from calculations according to a first principle procedure. The agreement with the model parameters issued from experimental EPR data is good. The spin and orbital contributions to the model parameters are evaluated. Calculations are compared to crystal field theory at the different steps of calculations: while this theory is a very good framework to explain the main magnetic behavior, it is shown that it is not able to render precisely all the small effects.
\end{abstract}

\section{Introduction}

Magnetic properties of actinide compounds are relatively unexplored due to the difficulties of experimental work. For lanthanides, the magnetic properties can be usually analyzed as a small perturbation of the free ion properties by the crystal field (CF) created by the environment since the 4 forbitals are well shielded by the closed shell $5 s$ and $5 p$ orbitals. In actinide complexes, the interaction of the ion with the environment is much larger and leads to novel properties since the spin-orbit (SO) coupling is important in these compounds. Most of the work in this field deals with the magnetic properties in the solid state. Relatively little work has been done in molecular chemistry until recently. Molecular magnetism of U(III) complexes arouses interest due to their Single Molecule Magnets properties. These molecules have complex structures and quantum chemical studies have shown to provide information complementary to experiment $[1,2]$. In the 50s and 60s, many EPR experiments were performed on diluted actinide ions in highly symmetric nonmagnetic crystals [3]. These magnetic data can be modeled by spin Hamiltonians and due to the high symmetry of the CF environment of the paramagnetic ion, EPR spectra can be rationalized by the use of few model parameters. The complexes with $5 f^{1}$ configuration are easy to interpret since there is no need to build any many-electron wave functions. The $5 f^{1} \mathrm{AnX}_{6}^{q-}$ series has been well determined experimentally and has served as benchmark for quantum chemical methods: either based on wave function theory $[4,5]$

\footnotetext{
* Corresponding author. Tel.: +33 561556467.

E-mail address: bolvin@irsamc.ups-tlse.fr (H. Bolvin).
}

or on Density Functional Theory [6-8]. A recent study of the $\mathrm{NpO}_{2}^{2+}$ cation with different equatorial ligands has confirmed the capability of these methods to calculate the $\mathrm{g}$ factors of both ground and excited states [33]. In these studies, it was shown that CF theory permits to model accurately the computed properties of these highly symmetrical $5 f^{1}$ complexes. A very recent study [9] applies this theory in order to analyze the bonding scheme in $\mathrm{U}(\mathrm{V})$ complexes. We should also mention the work of Seijo and Barandiaran [10] who calculated the spectrum of excited states of $\mathrm{Pa}^{4+}$ in the $\mathrm{Cs}_{2} \mathrm{ZrCl}_{6}$ crystal. The aim of this work is to calculate the magnetic properties of a symmetrical $5 f^{3}$ complex and to discuss the results in the light of CF theory.

We present calculations from first principles of the magnetic properties of a $5 f^{3}$ complex in an octahedral environment: $\mathrm{Np}^{4+}$ diluted in $\mathrm{Cs}_{2} \mathrm{ZrCl}_{6}$ crystal. A $5 f^{3}$ paramagnetic ion in an octahedral site has a fourfold degenerate ground state of symmetry $F_{3 / 2 u}$ according to Mulliken's notation [11] ( $\Gamma_{8}$ according to Bethe's notations [3]). This state can be split in two Kramers doublets due to geometric distortions of the environment. It seems to be the case in the $\mathrm{Cs}_{2} \mathrm{NpCl}_{6}$ crystal according to the measurement of magnetic susceptibility [12] and in the $\left(\mathrm{Ph}_{4} \mathrm{P}\right)_{2} \mathrm{NpCl}_{6}$ crystal where X-ray spectroscopy shows a distortion within the $\mathrm{NpCl}_{6}^{2-}$ cluster [13]. The magnetic properties of the $\mathrm{NpCl}_{6}^{2-}$ cluster diluted in the cubic $\mathrm{Cs}_{2} \mathrm{ZrCl}_{6}$ crystal have been characterized by EPR spectroscopy by Bray in 1978 [14]; Bray concludes to the presence of a ground quadruplet. Magnetic properties of ground states can be efficiently modeled by spin Hamiltonians; these model Hamiltonians act in a model spin space spanned by the $\left|\tilde{M}_{S}\right\rangle$ kets which are eigenvectors of the $\tilde{S}_{z}$ pseudospin operator. The value of the pseudospin $\tilde{S}$ and consequently the size of the model space is chosen according to the 
degeneracy of the ground state; in the case of a fourfold degeneracy, $\tilde{S}=3 / 2$. Bleaney and Abragam $[15,3]$ have shown that the spin Hamiltonian describing a $F_{3 / 2 u}$ state takes the form

$\hat{H}_{S}=\mu_{B} g \hat{\tilde{\boldsymbol{S}}} \cdot \boldsymbol{B}+\mu_{B} G\left(B_{x} \hat{\tilde{S}}_{x}^{3}+B_{y} \hat{\tilde{S}}_{y}^{3}+B_{z} \hat{\tilde{S}}_{z}^{3}\right)$

where $\mu_{B}$ is the Bohr magneton, $\boldsymbol{B}$ is the applied magnetic field and $g$ and $G$ are two scalar model parameters. The magnetic properties of the ground state are entirely defined by these two parameters $g$ and $G$.

The ground state of the free $\mathrm{Np}^{4+}$ ion is ${ }^{4} I_{9 / 2}$. The degeneracy of this term is removed by the $\mathrm{CF}$ of the octahedral environment in $2 F_{3 / 2 u} \oplus E_{1 / 2 u}$. For $f$ elements, the CF operator in a cubic point symmetry acting in a $J$ manifold can be written as

$$
\hat{V}_{C F}=\beta(J) A_{4}\left\langle r^{4}\right\rangle\left(\hat{O}_{4}^{0}(J)+5 \hat{O}_{4}^{4}(J)\right)+\gamma(J) A_{6}\left\langle r^{6}\right\rangle\left(\hat{O}_{6}^{0}(J)-21 \hat{O}_{6}^{4}(J)\right)
$$

where $\hat{O}_{k}^{q}$ are the Stevens operators, $A_{k}\left\langle r^{k}\right\rangle$ the CF parameters, and $\beta(J)$ and $\gamma(J)$ the parameters issued from the Wigner-Eckart theorem, depending on the $f^{n}$ configuration and on the representation used to determine the state [16]. Lea, Leask and Wolf (LLW) have rewritten this operator on the following form [17]

$\hat{V}_{C F}=W\left[x \frac{\hat{O}_{4}}{F(4)}+(1-|x|) \frac{\hat{O}_{6}}{F(6)}\right]$

with $\hat{O}_{4}=\hat{O}_{4}^{0}(J)+5 \hat{O}_{4}^{4}(J)$ and $\hat{O}_{6}=\hat{O}_{6}^{0}(J)-21 \hat{O}_{6}^{4}(J) . F(4)$ and $F(6)$ are factors dependent on the value of $J$. There are two independent CF parameters for $f$ elements in a cubic field. In Eq. (2), these parameters are $A_{4}\left\langle r^{4}\right\rangle$ and $A_{6}\left\langle r^{6}\right\rangle$; they are independent of the chosen representation and are characteristic of the compound. In Eq. (3), the two parameters are $W$ and $x$; they depend on the $J$ manifold but are useful since $x$ characterizes the ratio between 4 th and 6th order CF operators while $W$ plays the role of a scaling parameter. LLW have plotted the energies of all the states issued from a J manifold according to $x$. In general, $x$ determines the nature of the ground state for a given sign of $W$. In the case of $J=9 / 2$, since there are two $F_{3 / 2 u}$ states in this manifold, $x$ determines the ratio between these two states and consequently determines the magnetic properties as shown in Fig. 1 and in Appendix 2.

The first fitting of the EPR spectrum of $\mathrm{Np}^{4+}$ diluted in $\mathrm{Cs}_{2} \mathrm{ZrCl}_{6}$ by Bray led to $x=-0.567, g=0.40$ and $G=-1.7$ [14]. These results were startling since $x$ should be positive in the case of an octahedral CF. One year later, Bernstein and Dennis fitted the experimental data using the full information about the dependency on the orientation of the magnetic field; this new analysis led to new values for the parameters $g=-0.516, G=0.882$ and $x=0.475$ [18]. It shows that in some cases, the extraction of model parameters from experimental data is not obvious and can lead to erroneous results.
The aim of this article is a first principle description of the magnetic properties of the ground state of the $\mathrm{NpCl}_{6}^{2-}$ cluster diluted in $\mathrm{Cs}_{2} \mathrm{ZrCl}_{6}$. The SO-CASPT2 method has been chosen since it has successfully reproduced the $\mathrm{g}$ factors in the case of octahedral actinide compounds with twofold degenerate ground states and, using the properties of symmetry, allowed to deduce the sign of the $g$ factors from first principle arguments [19]. In the first section we describe the computational details of the calculations. Then we present the results, compare them to the available experimental data and discuss them in the light of CF theory. Both CF and spin Hamiltonian parameters are calculated from the computed observables.

\section{Computational details}

Calculations have been performed using the MOLCAS-78 suite of programs [20]. The active space consists of three electrons in the seven 5 f orbitals of the Np atom. First, a SF-CASSCF (Spin-Free Complete Active Space Self Consistent Field) calculation is performed [21] with 35 and 84 roots for spin quartets and doublets respectively. This first step provides the zeroth order multiconfigurational wave function without spin-orbit. Dynamical correlation is added using the SF-CASPT2 (Spin-Free Complete Active Space Perturbation Theory at 2nd order) method [22] without any level shift. This step includes the correlation of the movement of the electrons. The corresponding states at the SF-CASSCF and SF-CASPT2 levels span irreducible representations of the simple point group. SO coupling is included by a state interaction between the SF-CASSCF wave functions by the RASSI (Restricted Active Space State Interaction) method [23]; one gets the SO-CASSCF results which include the SO but not the dynamical correlation effects. In order to combine SO and dynamical correlation, the SO matrix is dressed with the CASPT2 energies giving rise to the SO-CASPT2 method. Both the SO-CASSCF and SO-CASPT2 methods provide states spanned by the irreducible representations of the double group. Calculations have been performed without any symmetry restriction. Scalar relativistic effects are taken into account by means of the Douglas-Kroll-Hess transformation [24] and SO integrals are calculated using the AMFI (Atomic Mean-Field Integrals) approximation [25]. The cluster $\mathrm{NpCl}_{6}^{2-}$ is described by all-electron basis sets ANORCC of TZP quality (Np: 9s8p6d4f2g1h; Cl: 5s4p2d1f) [26,27]. The first shells of the $8 \mathrm{Cs}^{+}$cations, the $48 \mathrm{Cl}^{-}$anions and the $12 \mathrm{Zr}^{4+}$ cations are described by model potentials without basis sets [10]. The rest of the crystal is represented by 352 point charges. We have performed some calculations where the first shell of Cs cations is described using basis sets and the results were very similar to those presented in this work. Model potentials and point charges are positioned according to the $\mathrm{Cs}_{2} \mathrm{ZrCl}_{6}$ lattice [28]. The charges have been determined by a LOPROP analysis [34] of a B3LYP calculation on the $\mathrm{ZrCl}_{6}^{2-}$ ion; +1.30 for $\mathrm{Zr},-0.55$ for $\mathrm{Cl}$ and +1.00 for $\mathrm{Cs}$. When using the pure ionic charges $(\mathrm{Np}+4.00$ and $\mathrm{Cl}-1.00)$ the relative energy of the states varies within some 10 of $\mathrm{cm}^{-1}$. The $\mathrm{Np}-\mathrm{Cl}$ distance
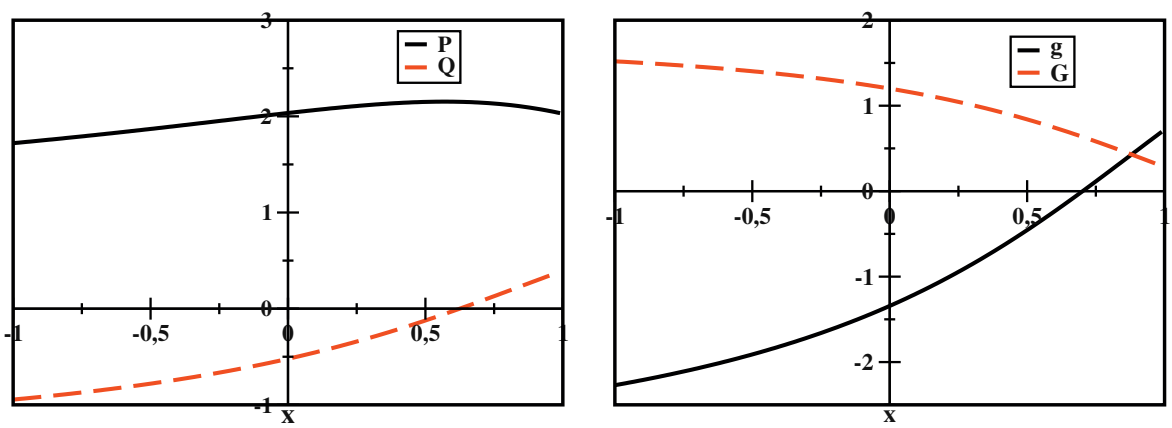

Fig. 1. $P$ and $Q$ in $\mu_{B}$ units (left) and $g$ and $G$ (right) as a function of $x$ for the lowest $F_{3 / 2 u}$ state in the frame of $C F$ theory. 
has been optimized by a DFT calculation using the ADF code [29]; the $\left[\mathrm{NpCl}_{6} \mathrm{Cs}_{8}\right]^{6+}$ cluster was described by TZ2P basis sets [30] and the rest by point charges. Only the $\mathrm{Np}-\mathrm{Cl}$ distance was optimized using the B3LYP functional [31] while the geometry of the rest of the crystal was kept fixed. A distance of $2.643 \AA$ was found, resulting in a slightly longer distance than the crystallographic $\mathrm{Zr}-\mathrm{Cl}$ one $(2.60175 \AA)$, as can be expected from ionic radius of $\mathrm{Np}^{4+}(0.87 \AA)$ larger than the $\mathrm{Zr}^{4+}$ one $(0.72 \AA)$. In the $\left(\mathrm{Ph}_{4} \mathrm{P}\right)_{2} \mathrm{NpCl}_{6}$ crystal, the average $\mathrm{Np}-\mathrm{Cl}$ distance is $2.61 \AA$. SO-CASPT2 calculations have been performed using both the optimized and the crystallographic distances. Matrix elements of orbital, spin and total angular momenta within the SO wave functions are determined by a local program using results of the RASSI code.

\section{Results and discussion}

The $\mathrm{Np}^{4+}$ ion is surrounded by six chloride anions in an octahedral environment. The next shell consists of $8 \mathrm{Cs}^{+}$cations. The structure is shown in Fig. 2. According to the calculations where the $\mathrm{Cs}^{+}$cations are described by basis sets, there is no charge transfer between the $\mathrm{NpCl}_{6}^{2-}$ cluster and the $\mathrm{Cs}^{+}$cations. The $5 f$ orbitals of the neptunium cation are split according to the $a_{2 u} \oplus t_{2 u} \oplus t_{1 u}$ scheme, as expected by CF theory. From the canonical energies of these orbitals for the multistate CASSCF calculation, the $\Delta=t_{2 u}-a_{2 u}$ and $\Theta=t_{1 u}-t_{2 u}$ energy gaps have been evaluated as 988 and $1448 \mathrm{~cm}^{-1}$, respectively. They correspond to the CF parameters $A_{4}<r^{4}>=593 \mathrm{~cm}^{-1}$ and $A_{6}<r^{6}>=29 \mathrm{~cm}^{-1}$; in Ref. [4], octahedral $5 f^{1}$ complexes were considered and for example, in $\mathrm{PaCl}_{6}^{2-}$, which has a similar metal ligand distance (2.64 $\AA$ ), $\Delta$ and $\Theta$ were found to be equal to 1390 and $2430 \mathrm{~cm}^{-1}$ respectively. In the present case, $\mathrm{Np}^{4+}$ has a $5 f^{3}$ configuration and most of the SF states are highly multideterminantal. The multistate CASPT2 step mixes SF roots, such that it is difficult to analyze the final states in term of orbitals. The state scheme is represented in Fig. 3. The SF states issued from the ground SF free ion term ${ }^{4} I$ order at SF-CASSCF level according to ${ }^{4} A_{2 u},{ }^{4} T_{2 u},{ }^{4} E_{u},{ }^{4} T_{1 u},{ }^{4} A_{1 u},{ }^{4} T_{2 u}$ (see Table 1 ). This ordering of the states corresponds exactly to the splitting of a $L=6$ manifold by an octahedral environment as shown by LLW [17]. One retrieves the states of Fig. 5 of Ref. [17] with a negative value of $W^{\prime}$ and for $x^{\prime}$ in the range 0.4-0.5. We have determined the CF parameters from the energies of these six SF states by a

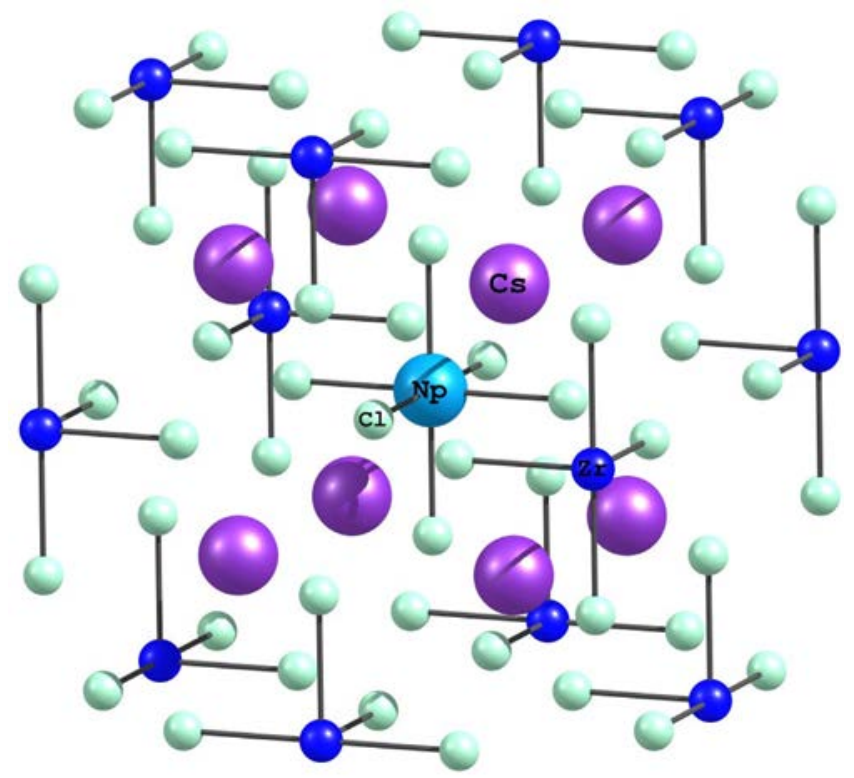

Fig. 2. The $\mathrm{NpCl}_{6}^{2-}$ cluster and the first shell of ions described by ECPs.

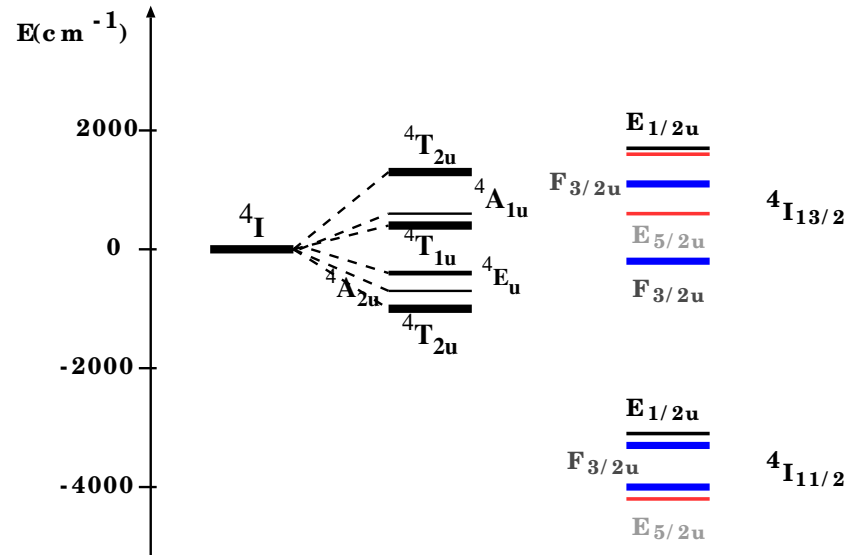

SF-CASPT2

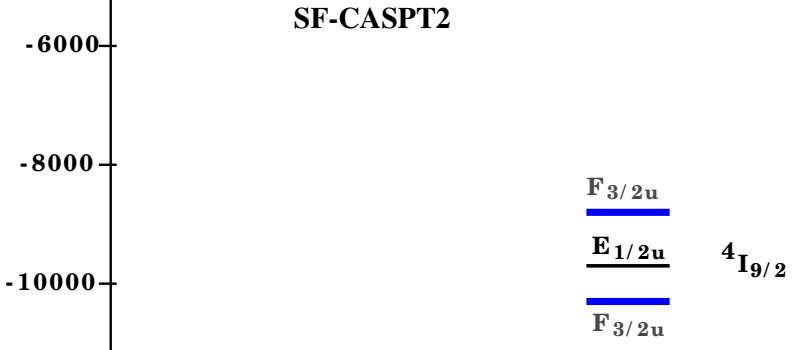

SO-CASPT2

Fig. 3. States of the $\mathrm{NpCl}_{6}^{26}$ cluster at the CASPT2 level for a Np-Cl distance of $2.643 \mathrm{~A}$.

Table 1

Calculated energy gaps and model parameters compared to the experimental data. All energies are in $\mathrm{cm}^{-1}$.

\begin{tabular}{lllll}
\hline $\mathrm{d}_{N p-C l}(\AA)$ & 2.643 & & 2.60175 & exp \\
\cline { 2 - 4 } & SF-CASSCF & SF-CASPT2 & SF-CASPT2 & \\
\hline${ }^{4} A_{2 u}$ & 0 & 320 & 283 & \\
${ }^{4} T_{2 u}$ & 614 & 0 & 0 & \\
${ }^{4} E_{u}$ & 1133 & 761 & 801 & \\
${ }^{4} T_{1 u}$ & 1919 & 1537 & 1634 & \\
${ }^{4} A_{1 u}$ & 1979 & 1548 & 1647 & \\
${ }^{4} T_{2 u}(2)$ & 2247 & 2348 & 2491 & \\
$x^{\prime b}$ & 0.47 & 0.44 & 0.43 & \\
$W^{\prime}$ & -11 & -12 & -13 & \\
$\mathrm{rms}^{\mathrm{b}}$ & 50 & 273 & 277 & \\
$A_{4}<r^{4}>$ & 735 & 733 & 782 & \\
$A_{6}<r^{6}>$ & 69 & 82 & 88 &
\end{tabular}

\begin{tabular}{llllll} 
& SO-CASSCF & SO-CASPT2 & SO-CASPT2 $Q^{\mathrm{a}}$ & SO-CASPT2 & exp \\
\hline$F_{3 / 2 u}$ & 0 & 0 & 0 & 0 & $0^{\mathrm{e}}$ \\
$E_{1 / 2 u}$ & 327 & 266 & 408 & 258 & 900 \\
$F_{3 / 2 u}(2)$ & 1094 & 1115 & 1258 & 1417 & 982 \\
$E_{5 / 2 u}$ & 5738 & 5980 & 4251 & 5730 & 5836 \\
$\chi^{\mathrm{c}}$ & 0.70 & 0.73 & 0.70 & 0.76 & \\
$W$ & -19 & -20 & -22 & -25 & \\
$A_{4}<r^{4}>$ & 784 & 833 & 884 & 1100 & \\
$A_{6}<r^{6}>$ & 59 & 55 & 70 & 64 & \\
$g$ & -0.182 & -0.406 & -0.362 & -0.411 & $-0.516^{\mathrm{f}}$ \\
$G$ & 0.657 & 0.785 & 0.712 & 0.779 & 0.882 \\
$G$ & 1.948 & 2.040 & 1.858 & 2.012 & 2.203 \\
$P\left(\mu_{B}\right)$ & -0.010 & -0.105 & -0.092 & -0.108 & -0.148 \\
$Q\left(\mu_{B}\right)$ & -0.63 & 0.51 & 0.51 & 0.51 & \\
$x^{\mathrm{d}}$ & 0.63 & 0.098 & 0.21 & 0.10 & \\
rms & 0.14 & & & & \\
\hline
\end{tabular}

a State interaction restricted to spin quartets.

b Deduced from a least square procedure from SF energy gaps.

c Deduced from SO energy gaps.

d Deduced from a least square procedure from $P(x)$ and $Q(x)$ in Eq. (18).

e Absorption spectrum of $\mathrm{Cs}_{2} \mathrm{NpCl}_{6}$ [32].

${ }^{f}$ From Ref. [18]. 
least square procedure (see Appendix 1). At the SF-CASPT2 level, we found $x^{\prime}=0.44, W=-12 \mathrm{~cm}^{-1}$ leading to $A_{4}<r^{4}>=733 \mathrm{~cm}^{-1}$ and $A_{6}<r^{6}>=82 \mathrm{~cm}^{-1}$. These values are quite different from the previous ones deduced from orbital considerations. The values deduced for SF-CASSCF are given in Table 1. It has to be pointed out that the SF-CASSCF levels are much better fitted by CF theory than the SFCASPT2 ones; the ordering of the states is not the same for the two levels of calculations, and more precisely the ground state is not the same: at the CASPT2 level, the ground state is a ${ }^{4} T_{2 u}$ state and there is no value of $x^{\prime}$ that predicts this state to be the ground one. Consequently, the rms (root mean square) is small for the SF-CASSCF level but becomes much larger at the SF-CASPT2 level.

The SO states are calculated by a state interaction between the SF states using the SO-RASSI code. The three first states of symmetry $F_{3 / 2 u} E_{1 / 2 u}$ and $F_{3 / 2 u}$ are well separated from the other ones by more than $4000 \mathrm{~cm}^{-1}$ : these states are those arising from the ${ }^{4} I_{9 / 2}$ term of the free ion. Again, the ordering corresponds to the one calculated by LLW for a $J=9 / 2$ manifold (see Appendix 1 ). $x$ and $W$ can be calculated from the energies of these three states (the reader is reminded that $x$ and $W$ depend on the considered manifold since they include the Wigner-Eckart coefficients $\beta$ and $\gamma$, see Appendix 1). Since in this case one models two energy gaps by two parameters, the rms is zero and the extraction of these parameters from first principles data is less significant than in the SF case. One finds $x=0.73$ and $W=-20 \mathrm{~cm}^{-1}$ leading to $A_{4}\left\langle r^{4}\right\rangle=833 \mathrm{~cm}^{-1}$ and $A_{6}\left\langle r^{6}\right\rangle=55 \mathrm{~cm}^{-1}$, values different but relatively close to the CF parameters deduced in the SF step. The extraction of $x$ from experimental data seems to be problematic. In Table 5 of Ref. [18], values of $-0.561,+0.475$ and 0.764 are given, depending on the fit. In the text, the authors conclude "Since $x=0.535$ and $x=0.453$ obtain for $\mathrm{Pa}^{4+}$ and $\mathrm{U}^{4+}$ respectively, the value $x=0.475$ for $\mathrm{Np}^{4+}$ seems more reasonable than does the value of $x=0.764$ ". This last sentence is not clear since the value of $x$ depends on the number of $f$ electrons and is not transferable from one ion to another.

The matrices of the total angular momentum operator components $\hat{M}_{x}, \hat{M}_{y}$ and $\hat{M}_{z}$ are calculated in the basis set $\left\{\left|\Psi_{a}^{1}\right\rangle,\left|\Psi_{b}^{1}\right\rangle,\left|\Psi_{c}^{1}\right\rangle,\left|\Psi_{d}^{1}\right\rangle\right\}$ of the kets issued from the SO-RASSI calculations. These wave functions have no specific symmetry properties. We are looking for the rotation within this space, such that the connection between the three matrices $\boldsymbol{M}_{x}, \boldsymbol{M}_{y}$ and $\boldsymbol{M}_{z}$ and the corresponding model matrices can be established. The spin Hamiltonian

$\hat{H}_{S}=\mu_{B} g \hat{\tilde{S}} \cdot \vec{B}+\mu_{B} G\left(B_{x} \hat{\tilde{S}}_{x}^{3}+B_{y} \hat{\tilde{S}}_{y}^{3}+B_{z} \hat{\tilde{S}}_{z}^{3}\right)=-\mu_{B} \hat{M}^{\bmod } \cdot \vec{B}$

leads to the following expression for the model magnetic momentum

$\hat{M}_{k}^{\text {mod }}=-g \hat{\tilde{S}}_{k}-G \hat{\tilde{S}}_{k}^{3}$

for $k=x, y, z$. In the basis set $\{|\tilde{3} / 2\rangle,|\tilde{1} / 2\rangle,|-\tilde{1} / 2\rangle,-|\tilde{3} / 2\rangle\}$ of the model space, the angular momentum matrices $\boldsymbol{M}_{x}^{\mathrm{mod}}, \boldsymbol{M}_{y}^{\mathrm{mod}}$ and $\boldsymbol{M}_{z}^{\text {mod }}$ take the following forms, in units of $\mu_{B}$

\begin{tabular}{c|cccc}
$\hat{M}_{x}^{\text {mod }}$ & $\left|\frac{\tilde{3}}{2}\right\rangle$ & $\left|\frac{\tilde{1}}{2}\right\rangle$ & $\left|-\frac{\tilde{1}}{2}\right\rangle$ & $\left|-\frac{\tilde{3}}{2}\right\rangle$ \\
\hline$\left\langle\frac{\tilde{3}}{2}\right.$ & 0 & $-\sqrt{3}\left(\frac{1}{2} g+\frac{7}{8} G\right)$ & 0 & $-\frac{3}{4} G$ \\
$\left\langle\tilde{\frac{1}{2}}\right.$ & $-\sqrt{3}\left(\frac{1}{2} g+\frac{7}{8} G\right)$ & 0 & $-\left(g+\frac{5}{2} G\right)$ & 0 \\
$\left\langle-\frac{1}{2}\right.$ & 0 & $-\left(g+\frac{5}{2} G\right)$ & 0 & $-\sqrt{3}\left(\frac{1}{2} g+\frac{7}{8} G\right)$ \\
$-\frac{\tilde{3}}{2} \mid$ & $-\frac{3}{4} G$ & 0 & $-\sqrt{3}\left(\frac{1}{2} g+\frac{7}{8} G\right)$ & 0
\end{tabular}

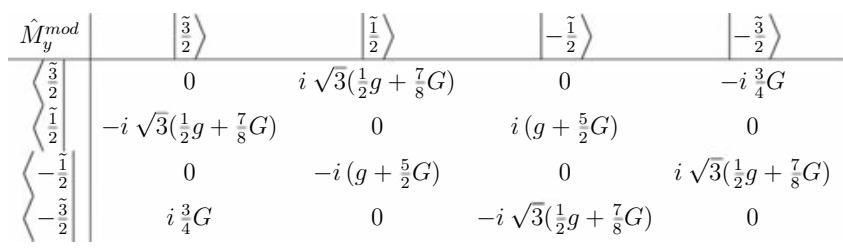

\begin{tabular}{c|ccc|c}
$\hat{M}_{z}^{\text {mod }}$ & $\left|\frac{\tilde{3}}{2}\right\rangle$ & $\left|\frac{\tilde{1}}{2}\right\rangle$ & $\left.-\frac{\tilde{1}}{2}\right\rangle$ & $\left|-\frac{\tilde{3}}{2}\right\rangle$ \\
\hline$\left\langle\frac{\tilde{3}}{2}\right|$ & $-\frac{3}{2} g-\frac{27}{8} G$ & 0 & 0 & 0 \\
$\left\langle\tilde{\frac{1}{2}}\right|$ & 0 & $-\frac{1}{2} g-\frac{1}{8} G$ & 0 & 0 \\
$\left\langle\begin{array}{c}\tilde{\frac{1}{2}} \\
\left\langle-\frac{\tilde{3}}{2}\right.\end{array}\right|$ & 0 & 0 & $\frac{1}{2} g+\frac{1}{8} G$ & 0 \\
0 & 0 & 0 & $\frac{3}{2} g+\frac{27}{8} G$
\end{tabular}

In the case of twofold degenerate states, the connection between the "physical" space and the model one is done by analyzing the properties of rotation of the spin operator [19]. We propose a procedure to build the kets $\left\{\left|\Psi_{3 / 2}\right\rangle,\left|\Psi_{1 / 2}\right\rangle,\left|\Psi_{-1 / 2}\right\rangle,\left|\Psi_{-3 / 2}\right\rangle\right\}$ as a rotation of the initial basis set $\left\{\left|\Psi_{a}^{1}\right\rangle,\left|\Psi_{b}^{1}\right\rangle,\left|\Psi_{c}^{1}\right\rangle,\left|\Psi_{d}^{1}\right\rangle\right\}$ in Appendix 3. As already pointed out by Bleaney [15], there are two equivalent solutions. The matrices $\boldsymbol{M}_{x}, \boldsymbol{M}_{y}$ and $\boldsymbol{M}_{z}$ expressed in this new basis set can be connected to the model matrices of Eqs. (6)-(8) such that $g$ and $G$ can be determined by a least square procedure. Results are summarized in Table 1 . The values of $P=-\left\langle\Psi_{3 / 2}\left|\hat{M}_{z}\right| \Psi_{3 / 2}\right\rangle \equiv$ $-\left\langle\tilde{3} / 2\left|\hat{M}_{z}^{\bmod }\right| \tilde{3} / 2\right\rangle$ and $Q=-\left\langle\Psi_{1 / / 2}\left|\hat{M}_{z}\right| \Psi_{1 / / 2}\right\rangle \equiv-\left\langle\tilde{1} / 2\left|\hat{M}_{z}^{\bmod }\right| \tilde{1} / 2\right\rangle$ are given. We focus on one of the solutions, since the other one can be obtained by a simple transformation (see Appendix 3). The values of $x$ can be deduced from $P(x)$ and $Q(x)$ given in Eq. (18): we found $x=0.51$ a value very different from the one deduced from the energy gaps. It should be pointed out that $Q=0.11$ for $x=0.73$; it shows that for the value of $x$ deduced from the energy gaps, $Q$ has the wrong sign and the magnetic properties are poorly reproduced. It means that we are not able to deduce $C F$ parameters that reproduce correctly both energy gaps and spin Hamiltonian parameters.

The values of $g$ and $G$ calculated from SO-CASPT2 results are closer to experiment than the SO-CASSCF ones; the dynamical correlation thus plays an important role. It changes the nature of SF ground state from ${ }^{4} A_{2 u}$ to ${ }^{4} T_{2 u}$ and the composition of the ground state in terms of SF states. The SO-CASSCF wave function is $39.0 \%$ ${ }^{4} T_{2 u}, 21.5 \%{ }^{4} A_{2 u} 14.3 \%{ }^{4} E_{u}$ while the SO-CASPT2 one is $38.3 \%{ }^{4} T_{2 u}$, $17.5 \%{ }^{4} A_{2 u} 13.8 \%{ }^{4} E_{u}$. The change in the composition of the wave function must be the origin of the change in the magnetic properties. The doublets contribute for $17 \%$ in the ground wave function; their effect is evaluated by making a state interaction restricted to the quartet states (3rd column of Table 1). One can see that their impact on the magnetic properties is quite small, but their effect on the energy gap between the ground $F_{3 / 2 u}$ and first excited state $E_{1 / 2 u}$ is more important. The comparison of the results for the optimized distance with the crystallographic one shows that the effect of the distance is small. The results for the shortest $\mathrm{Np}-\mathrm{Cl}$ distance are closer to the experimental ones. Menzel and Gruber [32] have measured the absorption spectrum of the $\mathrm{Cs}_{2} \mathrm{NpCl}_{6}$ crystal. The energy gap for the $F_{3 / 2 u} \rightarrow E_{1 / 2 u}$ transition is much higher than the one we find. Actually, they have observed many low lying bands $(58 ; 117$; $\left.128 ; 182 ; 260 ; 331 ; 900 ; 966 \mathrm{~cm}^{-1}\right)$. We propose that the two bands at 900 and $966 \mathrm{~cm}^{-1}$ arise from the splitting of the $F_{3 / 2 u}$ states due to the lowering of symmetry of the $\mathrm{NpCl}_{6}^{2-}$ that was not evidenced at the time of the experiment and that the band corresponding to the $F_{3 / 2 u} \rightarrow E_{1 / 2 u}$ transition is either at 260 or/and $331 \mathrm{~cm}^{-1}$ bands. 
Table 2

Spin and orbital contributions to the model parameters (SO-CASPT2 results for a $\mathrm{Np}-\mathrm{Cl}$ distance of $2.643 \AA$ )

\begin{tabular}{lrrr}
\hline & \multicolumn{1}{c}{ Total } & \multicolumn{1}{c}{ Spin } & Orbital \\
\hline$g$ & -0.406 & 0.027 & -0.460 \\
$G$ & 0.785 & -0.250 & 1.285 \\
$P\left(\mu_{B}\right)$ & 2.040 & -0.803 & 3.647 \\
$Q\left(\mu_{B}\right)$ & -0.105 & -0.017 & -0.069 \\
\hline
\end{tabular}

The matrices of spin and orbital momenta have been calculated in the $\left\{\left|\Psi_{3 / 2}\right\rangle,\left|\Psi_{1 / 2}\right\rangle,\left|\Psi_{-1 / 2}\right\rangle,\left|\Psi_{-3 / 2}\right\rangle\right\}$ basis set. These matrices have exactly the shape of Eqs. (6)-(8) such these contributions can be modeled by the spin Hamiltonian of Eq. (4) with model parameters $g_{S}, G_{S}$ and $g_{L}, G_{L}$ respectively. The results are reported in Table 2. It can be checked that $g_{L}+2 g_{S}=g$ and $G_{L}+2 G_{S}=G$ as expected. The orbital contribution is the largest and is opposite in sign to the spin one except in the case of $Q$ where both contributions have negative signs. The opposite sign and the largest orbital contribution can be understood from the free ion properties: in the ground term ${ }^{4} I_{9 / 2}$, $L$ and $S$ have opposite signs and $L$ is the largest.

\section{Conclusion}

In this work, we have presented the first ab initio calculations of magnetic properties of a fourfold degenerate state. In order to compare the computed magnetic momenta to the experimental ones modeled by a spin Hamiltonian, a rotation within the physical space generated by the four wave functions describing the ground state has to be done. In the new basis set, magnetic momentum matrices can be matched with those of the model Hamiltonian in the model space and spin Hamiltonian parameters can be calculated from first principles. We have proposed a procedure to achieve this point. The model parameters calculated in this manner compare well with the experimental ones. It should be noticed that calculation of the model parameters from experimental data is not obvious. The first set of model parameters proposed by Bray [14] was wrong. Then, the parameters proposed by Bernstein and Dennis were performed using figures taken from previous publications. The fitting of the data was not as good for $\mathrm{Np}^{4+}$ as expected "probably due to an internal inconsistency in the experimental data". They conclude "Finally, we wish to emphasize that the $\Gamma_{8}$ spin Hamiltonian parameters reported in Table II for $\mathrm{Np}^{4+} / \mathrm{Cs}_{2} \mathrm{ZrCl}_{6}$ are probably accurate only to within about $\pm 5 \%$." Our calculations have permitted to calculate the spin and orbital contributions to spin parameters; as can be expected from the respective values of $L$ and $S$ in the ground term of the free ion, spin and orbital contributions have opposite signs and the orbital one is the largest.

At each step of the calculation, CF parameters have been calculated from first principle data. The variation of the CF parameters depending on the observables they have been extracted from is important. It can be due to the fact that the calculation giving values of $g$ and $G$ closest to the experimental ones is the SO-CASPT2 one including the effect of more than 80 doublet states. Both the inclusion of dynamical correlation and the interaction with the excited SF spin doublet states influence the numerical results and these two effects are not taken into account by CF theory. It is important to emphasize, that CF theory provides a framework that permits to rationalize and understand the results. Though, the only exceptions in this work is the nature of the ground SF state at SF-CASPT2 level and the sign of $Q$ if $C F$ parameters deduced on energetic criteria is used. Otherwise, CF theory provides the correct ordering of states. But this work shows that quantitative analysis of numerical results is not possible in the framework of this theory.
Finally, this work confirms that the SO-CASPT2 approach is a valuable tool for the description of magnetic properties of actinide complexes. On the one hand, it reproduces well the available experimental data and on the other hand it provides access to properties that are not accessible by the experiment.

\section{Acknowledgments}

This work was supported by the ANR Grant ANR-09-BLAN-0195 TEMAMA. The authors would like to acknowledge very interesting discussions with Liviu Chibotaru and Liviu Ungur from the Katholieke Universiteit in Leuven, Belgium.

\section{Appendix 1. Crystal field equations according to LLW scheme}

In a $1=3$ manifold

$$
\begin{aligned}
& \beta(l)=\frac{2}{495}, \quad \gamma(l)=-\frac{4}{3861} \\
& E\left(a_{2 u}\right)=-48 W^{\prime \prime} \\
& E\left(t_{1 u}\right)=4 W^{\prime \prime}\left(-5+11 x^{\prime \prime}\right) \\
& E\left(t_{2 u}\right)=-4 W^{\prime \prime}\left(-9+11 x^{\prime \prime}\right) \\
& \text { In } a L=6 \text { manifold } \\
& \quad \beta(L)=-\frac{2}{16335}, \quad \gamma(L)=-\frac{10}{891891} \\
& E\left(A_{1}\right)=-2 W^{\prime}\left(-8+71 x^{\prime}\right) \\
& E\left(A_{2}\right)=-22 W^{\prime}\left(-8+5 x^{\prime}\right) \\
& E(E)=6 W^{\prime}\left(-8+27 x^{\prime}\right) \\
& E\left(T_{1}\right)=-8 W^{\prime}\left(-1+13 x^{\prime}\right) \\
& E\left(a T_{2}\right)=W^{\prime}\left(-5+10 x^{\prime}+\sqrt{784-1442 x^{\prime}+1079 x^{\prime 2}}\right) \\
& E\left(b T_{2}\right)=W^{\prime}\left(-5+10 x^{\prime}-\right.
\end{aligned}
$$

In a $J=9 / 2$ manifold

$$
\begin{aligned}
\beta(J) & =-\frac{136}{467181}, \quad \gamma(J)=-\frac{1615}{42513471} \\
E\left(E_{1 / 2}\right) & =\frac{4}{5} W(-80+129 x) \\
E\left(a F_{3 / 2}\right) & =\frac{1}{5} W\left(80-129 x-5 \quad \overline{1360-1152 x+513 x^{2}}\right) \\
E\left(b F_{3 / 2}\right) & =\text { frac } 15 W / 5\left(80-129 x+5 \quad \overline{1360-1152 x+513 x^{2}}\right)
\end{aligned}
$$

\section{Comparison of CF and LLW schemes}

By comparing Eqs. (6)-(8), one obtains

$A_{4}^{0}<r^{4}>=W x /(\beta F(4))$ and $A_{6}^{0}<r^{6}>=W(1-x) /(\gamma F(6))$

For example, $A_{4}<r^{4}>=800 \mathrm{~cm}^{-1}$ and $A_{6}<r^{6}>=88 \mathrm{~cm}^{-1}$ lead to

- $x^{\prime \prime}=1.51$ and $W^{\prime \prime}=32 \mathrm{~cm}^{-1}$ in the $l=3$ manifold .

- $x^{\prime}=0.44$ and $W^{\prime}=-13 \mathrm{~cm}^{-1}$ in the $L=6$ manifold.

- $x=0.62$ and $W=-22 \mathrm{~cm}^{-1}$ in the $J=9 / 2$ manifold.

\section{Appendix 2. Magnetic properties in the $F_{3 / 2 u}$ state}

The free ion ground state is characterized by the following quantum numbers: $L=6, S=3 / 2, J=9 / 2$ and $g_{J}=8 / 11$ 
The composition of each $M_{J}$ state is obtained from the Clebsch-Gordan coefficients $\left|M_{J}\right\rangle=\sum_{M_{L}=-L}^{L} \sum_{M_{S}=-S}^{S}\left\langle L M_{L} S M_{S}\right.$ $\left|S M_{J}\right\rangle\left|M_{L} M_{S}\right\rangle$.

In the basis set of the $\left|M_{J}\right\rangle$, the two operators $1 / F(4) \hat{O}_{4}$ and $1 / F(6) \hat{O}_{6}$ give rise to the following matrices
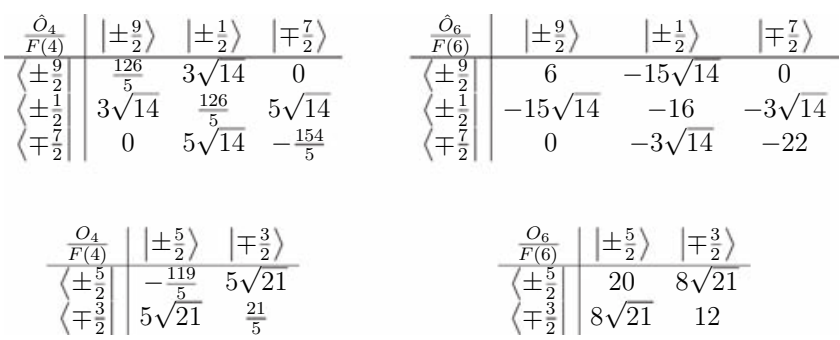

The diagonalization of $\hat{V}_{C F}=W\left[x O_{4} / F(4)+(1-|x|) O_{6} / F(6)\right]$ leads to three states of energies given by Eq. (11). In the region $x<0.83$ and for $W<0$, the ground state is the fourfold degenerate $b F_{3 / 2}$ one. The corresponding eigenvectors are

$$
\begin{aligned}
& \left|\Psi_{1}\right\rangle=a(x)\left|\frac{9}{2}\right\rangle+b(x)\left|\frac{1}{2}\right\rangle+c(x)\left|-\frac{7}{2}\right\rangle \\
& \left|\Psi_{2}\right\rangle=a(x)\left|-\frac{9}{2}\right\rangle+b(x)\left|-\frac{1}{2}\right\rangle+c(x)\left|\frac{7}{2}\right\rangle \\
& \left|\Psi_{3}\right\rangle=d(x)\left|\frac{5}{2}\right\rangle+e(x)\left|-\frac{3}{2}\right\rangle \\
& \left|\Psi_{4}\right\rangle=d(x)\left|-\frac{5}{2}\right\rangle+e(x)\left|\frac{3}{2}\right\rangle
\end{aligned}
$$

with

$$
\begin{aligned}
& a(x)=-\left(35-9 x+\sqrt{1360-1152 x+513 x^{2}}\right) /(3 N) \\
& b(x)=\left(38-17 x+\overline{1360-1152 x+513 * x^{2}}\right) /(\sqrt{14} N) \\
& c(x)=(-3+8 x) / N \\
& d(x)=-\left(4-18 x+\overline{1360-1152 x+513 x^{2}}\right) /\left(\sqrt{21} N^{\prime}\right) \\
& e(x)=(-8+3 x) / N^{\prime}
\end{aligned}
$$

One deduces the matrices for the three $\hat{M}_{u}(u=x, y, z)$ operators

\begin{tabular}{c|cccc}
$\hat{M}_{x}$ & $\left|\Psi_{2}\right\rangle$ & $\left|\Psi_{3}\right\rangle$ & $\left|\Psi_{4}\right\rangle$ & $\left|\Psi_{1}\right\rangle$ \\
\hline$\left\langle\Psi_{2}\right|$ & 0 & $\frac{8 \sqrt{6}}{11} b e+\frac{16}{11} c d$ & 0 & $\frac{24}{11} a c+\frac{20}{11} b^{2}$ \\
$\left\langle\Psi_{3}\right|$ & $\frac{8 \sqrt{6}}{11} b e+\frac{16}{11} c d$ & 0 & $\frac{8 \sqrt{21}}{11} e d$ & 0 \\
$\left\langle\Psi_{4}\right|$ & 0 & $\frac{8 \sqrt{21}}{11} e d$ & 0 & $\frac{8 \sqrt{6}}{11} b e+\frac{16}{11} c d$ \\
$\left\langle\Psi_{1}\right|$ & $\frac{24}{11} a c+\frac{20}{11} b^{2}$ & 0 & $\frac{8 \sqrt{6}}{11} b e+\frac{16}{11} c d$ & 0
\end{tabular}

\begin{tabular}{c|cccc}
$\hat{M}_{y}$ & $\left|\Psi_{2}\right\rangle$ & $\left|\Psi_{3}\right\rangle$ & $\left|\Psi_{4}\right\rangle$ & $\left|\Psi_{1}\right\rangle$ \\
\hline$\left\langle\Psi_{2}\right|$ & 0 & $-i\left(\frac{8 \sqrt{6}}{11} b e+\frac{16}{11} c d\right)$ & 0 & $i\left(\frac{24}{11} a c+\frac{20}{11} b^{2}\right)$ \\
$\left\langle\Psi_{3}\right|$ & $i\left(\frac{8 \sqrt{6}}{11} b e+\frac{16}{11} c d\right)$ & 0 & $-i \frac{8 \sqrt{21}}{11} e d$ & 0 \\
$\left\langle\Psi_{4}\right|$ & 0 & $i \frac{8 \sqrt{21}}{11} e d$ & 0 & $-i\left(\frac{8 \sqrt{6}}{11} b e+\frac{16}{11} c d\right)$ \\
$\left\langle\Psi_{1}\right|$ & $-i\left(\frac{24}{11} a c+\frac{20}{11} b^{2}\right)$ & 0 & $i\left(\frac{8 \sqrt{6}}{11} b e+\frac{16}{11} c d\right)$ & 0
\end{tabular}

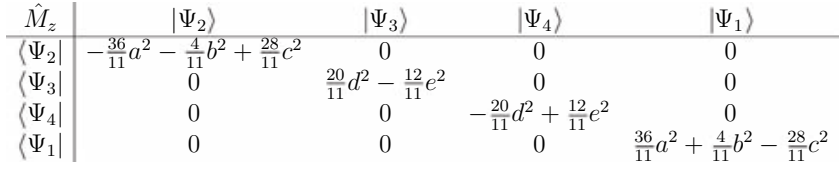

The comparison of these three matrices with Eqs. (21)-(23) results in two solutions for $P(x)$ and $Q(x)$

$$
\begin{aligned}
& P(x)=\frac{36}{11} a^{2}(x)+\frac{4}{11} b^{2}(x)-\frac{28}{11} c^{2}(x) \\
& Q(x)=-\frac{20}{11} d^{2}(x)+\frac{12}{11} e^{2}(x)
\end{aligned}
$$

or

$$
\begin{aligned}
& P(x)=\frac{20}{11} d^{2}(x)-\frac{12}{11} e^{2}(x) \\
& Q(x)=-\frac{36}{11} a^{2}(x)-\frac{4}{11} b^{2}(x)+\frac{28}{11} c^{2}(x)
\end{aligned}
$$

It should be noticed that

$$
\begin{aligned}
& \frac{\sqrt{3}}{4}\left(\frac{36}{11} a^{2}(x)+\frac{4}{11} b^{2}(x)-\frac{28}{11} c^{2}(x)-\frac{20}{11} d^{2}(x)+\frac{12}{11} e^{2}(x)\right) \\
& \quad=\frac{8 \sqrt{6}}{11} b(x) e(x)+\frac{16}{11} c(x) d(x) \\
& \frac{36}{11} a^{2}(x)+\frac{4}{11} b^{2}(x)-\frac{28}{11} c^{2}(x) \frac{20}{11} d^{2}(x)-\frac{12}{11} e^{2}(x) \\
& \quad=\frac{24}{11} a(x) c(x)+\frac{20}{11} b^{2}(x)
\end{aligned}
$$

It follows

$$
\begin{aligned}
& g(x)=-\frac{1}{12} P(x)+\frac{27}{12} Q(x) \\
& G(x)=\frac{1}{3} P(x)-Q(x)
\end{aligned}
$$

The first solution is represented in Fig. 1 since it is the solution matching the experimental data.

\section{Appendix 3. Spin Hamiltonian parameters from first principles calculations}

In the basis set $\{|\tilde{3} / 2\rangle,|\tilde{1} / 2\rangle,|-\tilde{1} / 2\rangle,|-\tilde{3} / 2\rangle\}$ of the model space, the angular momentum matrices $\boldsymbol{M}_{x}^{\mathrm{mod}}, \boldsymbol{M}_{y}^{\mathrm{mod}}$ and $\boldsymbol{M}_{z}^{\mathrm{mod}}$ take the forms of Eqs. (6)-(8). The SO-RASSI calculation provides a basis set $\left\{\left|\Psi_{a}^{1}\right\rangle,\left|\Psi_{b}^{1}\right\rangle,\left|\Psi_{c}^{1}\right\rangle,\left|\Psi_{d}^{1}\right\rangle\right\}$ in which the total angular matrices $\boldsymbol{M}_{x}, \boldsymbol{M}_{y}$ and $\boldsymbol{M}_{z}$ are calculated. We are looking for the rotation in the space generated by these wave functions such the three matrices $\boldsymbol{M}_{x}, \boldsymbol{M}_{y}$ and $\boldsymbol{M}_{z}$ have the shape of Eqs. (6)-(8). We have followed the following procedure: (i) one diagonalizes $\boldsymbol{M}_{z}$ and its eigenvectors give a new set $\left\{\left|\Psi_{a}^{2}\right\rangle,\left|\Psi_{b}^{2}\right\rangle,\left|\Psi_{c}^{2}\right\rangle,\left|\Psi_{d}^{2}\right\rangle\right\}$ with eigenvalues $\pm P$ and $\pm Q$. (ii) One determines the phase factors such $\boldsymbol{M}_{X}$ in the basis set $\left\{\left|\Psi_{1}^{3}\right\rangle=\left|\Psi_{1}^{2}\right\rangle,\right\}\left|\Psi_{2}^{3}\right\rangle=e^{i \alpha}\left|\Psi_{2}^{2}\right\rangle,\left|\Psi_{3}^{3}\right\rangle=e^{i \beta}\left|\Psi_{3}^{2}\right\rangle,\left|\Psi_{4}^{3}\right\rangle=$ $\left.e^{i \gamma}\left|\Psi_{4}^{2}\right\rangle\right\}$ becomes a real matrix. (iii) One finds the permutation such in basis set $\left.\pm\left|\Psi_{i}^{3}\right\rangle, \pm\left|\Psi_{j}^{3}\right\rangle, \pm\left|\Psi_{k}^{3}\right\rangle, \pm\left|\Psi_{l}^{3}\right\rangle\right\}, \quad \boldsymbol{M}_{x}, \quad \boldsymbol{M}_{y}$ and $\boldsymbol{M}_{z}$ can be written as in Eqs. (6)-(8). Actually, there are precisely two of them (except to a global change of phase factors of all the wave functions). Let us call $\left\{\left|\Psi_{3 / 2}\right\rangle,\left|\Psi_{1 / 2}\right\rangle,\left|\Psi_{-1 / 2}\right\rangle,\left|\Psi_{-3 / 2}\right\rangle\right\}$ 
one of them.

\begin{tabular}{c|cccc}
$\hat{M}_{x}$ & $\left|\Psi_{3 / 2}\right\rangle$ & $\left|\Psi_{1 / 2}\right\rangle$ & $\left|\Psi_{-1 / 2}\right\rangle$ & $\left|\Psi_{-3 / 2}\right\rangle$ \\
\hline$\left\langle\Psi_{3 / 2}\right|$ & 0 & $\frac{\sqrt{3}}{4}(P+Q)$ & 0 & $\frac{1}{4}(P-3 Q)$ \\
$\left\langle\Psi_{1 / 2}\right|$ & $\frac{\sqrt{3}}{4}(P+Q)$ & 0 & $\frac{1}{4}(3 P-Q)$ & 0 \\
$\left\langle\Psi_{-1 / 2}\right|$ & 0 & $\frac{1}{4}(3 P-Q)$ & 0 & $\frac{\sqrt{3}}{4}(P+Q)$ \\
$\left\langle\Psi_{-3 / 2}\right|$ & $\frac{1}{4}(P-3 Q)$ & 0 & $\frac{\sqrt{3}}{4}(P+Q)$ & 0
\end{tabular}

and

\begin{tabular}{c|cccc}
$\hat{M}_{y}$ & $\left|\Psi_{3 / 2}\right\rangle$ & $\left|\Psi_{1 / 2}\right\rangle$ & $\left|\Psi_{-1 / 2}\right\rangle$ & $\left|\Psi_{-3 / 2}\right\rangle$ \\
\hline$\left\langle\Psi_{3 / 2}\right|$ & 0 & $-i \frac{\sqrt{3}}{4}(P+Q)$ & 0 & $i \frac{1}{4}(P-3 Q)$ \\
$\left\langle\Psi_{1 / 2}\right|$ & $i \frac{\sqrt{3}}{4}(P+Q)$ & 0 & $-i \frac{1}{4}(3 P-Q)$ & 0 \\
$\left\langle\Psi_{-1 / 2}\right|$ & 0 & $i \frac{1}{4}(3 P-Q)$ & 0 & $-i \frac{\sqrt{3}}{4}(P+Q)$ \\
$\left\langle\Psi_{-3 / 2}\right|$ & $-i \frac{1}{4}(P-3 Q)$ & 0 & $i \frac{\sqrt{3}}{4}(P+Q)$ & 0
\end{tabular}

\begin{tabular}{c|cccc}
$\hat{M}_{z}$ & $\left|\Psi_{3 / 2}\right\rangle$ & $\left|\Psi_{1 / 2}\right\rangle$ & $\left|\Psi_{-1 / 2}\right\rangle$ & $\left|\Psi_{-3 / 2}\right\rangle$ \\
\hline$\left\langle\Psi_{3 / 2}\right.$ & $-P$ & 0 & 0 & 0 \\
$\Psi_{1 / 2} \mid$ & 0 & $-Q$ & 0 & 0 \\
$\left\langle\Psi_{-1 / 2}\right.$ & 0 & 0 & $Q$ & 0 \\
$\left\langle\Psi_{-3 / 2}\right.$ & 0 & 0 & 0 & $P$
\end{tabular}

In the final form, the matrix elements of $\boldsymbol{M}_{x}$ and $\boldsymbol{M}_{y}$ that are supposed to be zero are all lower than $10^{-3}$ (in units of $\left.\mu_{B}\right)$. The spin Hamiltonian parameters $g$ and $G$ are deduced from these matrices by a mean square procedure since there are two parameters for many matrix elements. The only permutation that preserves the correct forms of Eqs. (6) and (7) for $\boldsymbol{M}_{x}$ and $\boldsymbol{M}_{y}$ is $\left\{\Psi_{3 / 2} \leftrightarrow \Psi_{-1 / 2} ; \Psi_{-3 / 2} \leftrightarrow \Psi_{1 / 2}\right\}$ corresponding to $P^{\prime}=-Q$ and $Q^{\prime}=-P$. This leads to a set of new parameters $g^{\prime}=(-40 g-91 G) / 12$ and $G^{\prime}=(4 g+10 G) / 3$. The existence of these two solutions was already pointed out by Bleaney [15] and the two last equations can be found in his article.

We should point out that this procedure supposes that $\boldsymbol{M}_{x}$, $\boldsymbol{M}_{y}$ and $\boldsymbol{M}_{z}$ are calculated with $x, y$ and $z$ being the fourfold rotation axis of the octahedron. Since $x, y$ and $z$ are equivalent, a permutation between the three matrices leads to the same results. We have checked that first diagonalizing $\boldsymbol{M}_{x}$, then making $\boldsymbol{M}_{y}$ real leads to the same values for $g$ and $G$.

\section{References}

[1] J.T. Coutinho, M.A. Antunes, L.C.J. Pereira, H. Bolvin, J. Marçaloa, M. Mazzanti, M. Almeida, Dalton Trans. 41 (2012) 13568.

[2] M.A. Antunes, I.C. Santos, H. Bolvin, L.C.J. Pereira, M. Mazzanti, J. Marçaloa, M. Almeida, Dalton Trans. 42 (2013) 8861.

[3] A. Abragam, B. Bleaney, Electronic Paramagnetic Resonance of Transition Ions, Clarendon Press, Oxford, 1970.

[4] F.P. Notter, H. Bolvin, J. Chem. Phys. 130 (2009) 184310

[5] D. Ganyushin, F. Neese, J. Chem. Phys. 138 (2013) 104113.

[6] R. Arratia-Pérez, L. Hernandez-Acevedo, G.L. Malli, J. Chem. Phys. 121 (2004) 7743.

[7] R. Arratia-Pérez, G.L. Malli, J. Chem. Phys. 124 (2006) 074321.

[8] P. Verma, J. Autschbach, J. Chem. Theory Comput. 9 (2013) 1052.

[9] W.W. Lukens, N.M. Edelstein, N. Magnani, T.W. Hayton, S. Fortier, L.A. Seaman, J. Am. Chem. Soc. 135 (2013) 510742.

[10] L. Seijo, Z. Barandiaran, J. Chem. Phys. 115 (2001) 5554.

[11] S.L. Altmann, P. Herzig, Point-group Theory Tables, 2nd ed., Clarendon Press, Vienna, 2011.

[12] D.G. Karraker, A. Stone, Phys. Rev. B 22 (1980) 111.

[13] S.G. Minasian, K.S. Boland, R.K. Feller, S.A. Gaunt, A.J. Kozimor, I. May, S.D. Reilly, B.L. Scott, D.K. Shuh, Inorg. Chem. 51 (2012) 5728.

[14] J.E. Bray, Phys. Rev. B 18 (1978) 2978.

[15] B. Bleaney, Proc. Phys. Soc. Lond. 73 (1959) 939.

[16] K.W.H. Stevens, Proc. Phys. Soc., Lond., Sect. A 65 (1952) 209.

[17] K.R. Lea, M.J.M. Leask, W.P. Wolf, J. Phys. Chem. Solids 23 (1962) 1381

[18] E.R. Bernstein, L.W. Dennis, Phys. Rev. B 20 (1979) 870.

[19] L. Chibotaru, A. Ceulemans, H. Bolvin, Phys. Rev. Lett. 101 (2008) 033003.

[20] F. Aquilante, L. De Vico, N. Ferré, G. Ghigo, P.-Å. Malqvist, P. Neogrády, T.B. Pedersen, M. Pitonak, M. Reiher, B.O. Roos, M. Serrano-Andrés, M. Urban, V. Veryazov, R. Lindh, J. Comput. Chem. 31 (2010) 224.

[21] B.O. Roos, P.R. Taylor, P.E.M. Siegbahn, Chem. Phys. 48 (1980) 157.

[22] K. Andersson, P.-A. Malmqvist, B.O. Roos, A.J. Sadlej, K. Wolinski, J. Phys. Chem. 94 (1990) 5483.

[23] P.-Å. Malmqvist, B.O. Roos, B. Schimmelpfennig, Chem. Phys. Lett. 357 (2002) 230.

[24] B.A. Hess, Phys. Rev. A 33 (1986) 3742.

[25] B.A. Hess, C.M. Marian, U. Wahlgren, O. Gropen, Chem. Phys. Lett. 251 (1996) 365.

[26] B.O. Roos, R. Lindh, P.Å. Malmqvist, V. Veryazov, P.O. Widmark, J. Phys. Chem. A 108 (2004) 2851

[27] B.O. Roos, R. Lindh, P.Å. Malmqvist, V. Veryazov, P.O. Widmark, Chem. Phys. Lett. 409 (2005) 295

[28] R.W.G. Wyckoff, Crystal Structures, Wiley, New York, 1968.

[29] G. te Velde, F.M. Bickelhaupt, E.J. Baerends, C. Fonseca Guerra, S.J.A. van Gisbergen, J.G. Snijders, J. Comput. Chem. 22 (2007) 931.

[30] E. van Lenthe, E.J. Baerends, J. Comput. Chem. 24 (2003) 1142

[31] A.D. Becke, J. Chem. Phys. 98 (1993) 5648.

[32] E.R. Menzel, J.B. Gruber, J. Chem. Phys. 54 (1971) 3857.

[33] F. Gendron, D. Paez Hernandez, F. P. Notter, B. Pritchard, H. Bolvin, J. Autschbach Chem. Eur J. in press, 2011.

[34] L. Gagliardi, R. Lindh, G. Karlström, J. Chem. Phys. 121 (2004) 4494. 